\title{
EDUCACION EN CÁRCELES: PRÁCTICA ALIENANTE O LIBERADORA
}

\author{
EDUCATION IN JAILS: ALIENANT OR LIBERATING PRACTICE
}

EDUCACÃO EM PRISOES: PRÁTICA ALIENANTE OU LIBERADORA

BRAVO, Omar Alejandro1 ${ }^{\text {ID }}$

\section{RESUMEN}

Las prácticas educativas dentro de las cárceles pueden tener un rol alienante, en el sentido de inscribirse en un propósito resocializador, o un potencial liberador, en la medida en que favorezcan la reflexión en torno a las condiciones de vida de los sujetos y amplíe la posibilidad de transformarlas. Este artículo toma como referencia algunas de estas prácticas desarrolladas en la cárcel de Villahermosa, de la ciudad de Cali, Colombia, considerando en particular los textos que fundamentan el proyecto denominado Misión Carácter, y las analiza tomando como referencia al análisis institucional y el análisis de discurso. Este análisis se inscribe en una crítica general a los sistemas penitenciarios y su funcionalidad y muestra como estos discursos, de carácter moralista, refuerzan un instituido cuyas prácticas pretenden la conversión moral de los sujetos presos.

Palabras clave: Cárcel; educación. Análisis de discurso. Análisis institucional. Instituciones.

\section{ABSTRACT}

Educational practices within prisons can have an alienating role, in the sense of inscribing themselves in a resocializing purpose, or a liberating potential, insofar as they favor reflection on the living conditions of the subjects and expand the possibility to transform them. This article takes as reference some of these practices developed in the Villahermosa prison, in the city of Cali, Colombia, considering in particular the texts that underpin the project called Mission Character, and analyzes them taking as reference the institutional analysis and discourse analysis. This analysis is part of a general criticism of the penitentiary systems and their functionality and shows how these discourses, of a moralistic nature, reinforce an institutional practice that seeks the moral conversion of the prisoner.

Keywords: Jails. Education. Discourse analysis. Institutional analysis. Institutions.

\section{RESUMO}

As práticas educativas, dentro das prisões, podem ter um papel alienante, no sentido de se inscrever em um propósito resocializante, ou um potencial liberador, na medida em que favoreça a reflexão sobre as condições de vida dos sujeitos e amplie a possibilidade de transforma-las. Este artigo toma como referência algumas dessas práticas desenvolvidas na prisão de Villahermosa, da cidade de Cali, Colômbia, considerando particularmente os textos que fundamentam o projeto denominado Missão caráter, e as analisa tomando como referência à análise institucional e a análise de discurso. Esta análise se inscreve numa crítica geral aos sistemas penitenciários e sua funcionalidade e mostra como esses discursos, de caráter moralista, reforçam um instituído cujas práticas pretendem a conversão moral dos sujeitos presos.

Palavras-chave: Prisão. Educação. Analise de discurso. Analise institucional. Instituições.

\footnotetext{
1 Universidad Icesi - UI - Cali - Colombia
} 


\section{INTRODUCCIÓN}

Con frecuencia, el análisis del fenómeno carcelario se realiza a manera de juicio categórico, donde la crítica a estas instituciones suele apoyarse en Goffman (1970) y Foucault (1969; 1975; 2002), referencias ineludibles a este respecto.

El primero extiende su análisis a las llamadas instituciones totales, definidas como:

Lugar de residencia y trabajo, donde un gran número de individuos en igual situación, aislados de la sociedad por un periodo apreciable de tiempo, comparten en su encierro una rutina diaria administrada formalmente. (Goffman, 1970, p. 13).

Los efectos de las mismas, resumidos en la denominada mutilación del yo, dimensionan entonces las consecuencias de las rutinas impuestas, la pérdida de individualidad y el deterioro de la privacidad y la propia identidad. De esta forma, se impone la padronización agobiante de la vida sexual, los tiempos y espacios para dormir y comer, produciéndose así una muerte civil del sujeto.

Estas instituciones totales se pueden clasificar en aquellas dedicadas al cuidado de personas incapaces e inofensivas, como es el caso de los geriátricos; de protección de la comunidad, como los manicomios; de refugio del mundo exterior, siendo aquí ejemplos los monasterios, y las que se ocupan de encerrar a personas consideradas peligrosas, como las cárceles.

En lo que hace a Foucault, la dirección de su crítica apunta a mostrar la funcionalidad de las cárceles en relación al objetivo social de poder sostener espacios de reclusión y control a través de la producción de un supuesto enemigo social: el delincuente, que la propia prisión reproduce, sustentada también en un imaginario social que reclama castigo y aislamiento para ese Otro genérico y peligroso.

Ambas críticas resultan pertinentes. Los efectos de las cárceles, así como los de los manicomios, son atentatorios contra la salud mental de las personas que allí son recluidas y muestra una indisimulable paradoja entre los propósitos declarados de estas instituciones y sus efectos: intentan curar, pero patologizan; intentan resocializar, pero deterioran los vínculos laborales y familiares.

No obstante, también se pueden hacer algunas consideraciones generales sobre estos enfoques y su utilización. En primer lugar, cabe preguntarse los motivos por los cuales estos textos continúan siendo la referencia obligada para el análisis de estas instituciones, habiendo transcurrido cerca de cincuenta años desde su publicación.

En este sentido, y de forma especulativa, es posible hacer a este respecto una serie de preguntas: ¿las cárceles han cambiado muy poco desde la época en que Goffman y Foucault hicieron estas consideraciones? Sin duda puede afirmarse que hay una continuidad general de prácticas y discursos en las instituciones carcelarias, pero se debe señalar también la existencia creciente de 
formas de poder y regulación internas, hechas por los propios presos, que escapan en buena medida a esos análisis (Garland, 2206).

También en ese orden: el uso repetido de estos autores, ¿obedece, al menos en parte, a una insuficiente producción teórica en este campo? Puede afirmarse aquí que sí; en lo que hace al campo particular de la salud mental, por ejemplo, llama la atención la escasa reflexión existente en torno a los efectos subjetivos de las rutinas carcelarias, que escape a la perspectiva forense y habilite a un análisis categórico de los mismos (que, por ejemplo, lleve a negar la institución, en términos basaglianos).

No obstante estas consideraciones, el uso frecuente de las teorías de Goffman y Foucault en términos de juicio general, como ya fue mencionado, es indispensable y pertinente, pero no debe dejar de lado aspectos, en general de orden micro social, que hacen a la situación específica de las cárceles latinoamericanas y de cada prisión en particular.

Desde esta posible perspectiva ampliada de la institución carcelaria y las prácticas y discursos que la integran, cabe señalar la tensión constante entre estrategias y políticas de un supuesto fin resocializador, pero que en la práctica perpetúan formas de funcionamiento institucional, y formas de resistencia, más o menos explícitas que la propia comunidad carcelaria promueve o encuentra.

Este texto apunta a mostrar algunas de las prácticas y discursos señalados en primer término, tomando como referencia a la cárcel de Villahermosa, de la ciudad de Cali, Colombia y, en particular, al sector educativo de la misma, donde se dan clases de educación primaria y secundaria y se promueven también acciones dirigidas a la resocialización de las personas presas, extendiéndose también al público externo con un propósito preventivo.

\section{CAMPO EMPÍRICO}

Este establecimiento se ubica en el centro de la ciudad de Cali. Alberga a una población superior a las seis mil personas, siendo que su capacidad es de mil cien (INPEC, 2018). Solo está dirigida a hombres; las mujeres presas están en la cárcel situada en el municipio vecino de Jamundí, dentro de un complejo penitenciario de máxima seguridad construido con la intención de evitar el hacinamiento carcelario (pocos años después de inaugurado este espacio, ambos establecimientos se encuentran hoy desbordados en su capacidad).

La cárcel de Villahermosa se divide en diez patios o pabellones. Cuatro de ellos se sitúan en la denominada área externa: el patio destinado a personas de la tercera edad; el que aloja a los presos en fin de condena y que se ocupan de tareas generales, como la jardinería, por ejemplo; el patio originalmente destinado a integrantes de grupos paramilitares de derecha (aunque hoy en día, contiene a presos condenados o sindicados por otro tipo de crímenes) y el que está dirigido a funcionarios públicos.

Los patios internos se organizan alrededor de un extenso pasillo. Allí, las particularidades más marcadas, en cuanto a población, tienen que ver con el espacio destinado a perpetradores de crímenes 
sexuales; a miembros de pandillas del este de la ciudad, la mayoría joven y afrodescendiente y al sector reservado a población indígena e integrantes de grupos guerrilleros, existiendo también un área particular, más reciente, dirigido a la población LGBTI.

No obstante, tanto en estos patios mencionados como en los restantes, hay criterios particulares para la distribución de los presos, que expresan en parte las formas de poder que en este contexto se desarrollan.

Se conjugan aquí los mecanismos formales, propios de la institución, con los producidos por la propia población del penal. En cuanto a los primeros, al ingresar un preso nuevo al establecimiento, se lo aloja en un espacio fuera de los patios, hasta la realización de una entrevista, donde se lo indaga acerca de eventuales amistades y enemistades que pueda tener dentro de esa cárcel. Esta información, sumada a la del tipo de crimen cometido, la edad y la filiación laboral, como ya fue dicho, antecede al segundo criterio de ingreso y permanencia en un patio determinado.

El siguiente paso para acceder a esos espacios tiene que ver con la capacidad del sujeto para pagar los gastos requeridos, a manera de tasa de acceso y mensualidad, y los costos que se agregan, caso la persona desee tener una celda personal, por ejemplo. En este caso, es el grupo que manda en cada patio quien establece estos costos. Estas estructuras de poder son dirigidas por una persona, denominada "pluma", quien está respaldado por otros presos que sostienen esa posición por la fuerza, caso sea necesario. Eventualmente, se producen peleas por estos liderazgos, pero la forma de funcionamiento se mantiene.

Todo este proceso torna la vida en la cárcel, además de sufrida, cara. Las personas que no pueden pagar, están obligadas a vivir en los patios más congestionados y a dormir en el suelo.

Por otro lado, la rutina institucional implica desayunar muy temprano, previa extensa fila para acceder a los pocos y deteriorados baños, almorzar a media mañana y cenar al inicio de la tarde. Más allá de lo poco saludable de estos horarios, el hecho de comprimir las tres comidas en un lapso de tiempo tan corto, hace que aumente el ocio y el extenso tiempo libre, ya que estas actividades resumen el día a día de la mayoría de la población presa de Villahermosa.

Las opciones para trabajar y estudiar son también escasas. Para la primera, existen pocas posibilidades de trabajo y capacitación; en cuanto al estudio, el acceso a este espacio está sujeto a la buena conducta del interno, lo que también la limita. Entre los que pueden hacer parte del espacio educativo, hay también monitores, elegidos entre los propios internos, que se ocupan de dar apoyo a las actividades docentes y colaborar con el funcionamiento del sector.

Las áreas de la cárcel se completan con el precarizado sector de salud, a cargo de una empresa privada, y el de salud mental, que contiene a internos con problemas mentales significativos, psicóticos en la mayoría de los casos.

Para el funcionamiento de toda esta estructura, la cantidad de agentes penitenciarios es muy escasa. La gran dispersión sindical (existen decenas de representaciones gremiales de estos trabajadores) dificulta también negociar condiciones de trabajo más dignas. Por esto, esta labor es 
también productora de sufrimiento psíquico, lo que se percibe en la cantidad de trabajadores bajo tratamiento psiquiátrico y/o medicados.

En líneas generales, puede considerarse que esta cárcel representa lo que Wacquant (2009) denominó como aspiradora social, en el sentido de contener principalmente a

\footnotetext{
pequeños delincuentes ocasionales, desempleados e indigentes, sin techo e inmigrantes indocumentados, drogadictos, discapacitados y enfermos mentales, abandonados por el deterioro de la red de seguridad social y la salud, y jóvenes de las clases populares obligados a llevar una vida de empleos marginales y agobiados por la normalización del trabajo precario. ( $p$. 387).
}

La intervención en curso en el área educativa, a cargo de estudiantes y profesores del Departamento de Estudios Psicológicos de la universidad Icesi de Cali, se relaciona, en los últimos años, con la producción de una revista denominada Desde adentro. Esta publicación, como ya fue mencionado en otros artículos (Bravo, 2010; 2012; 2012ª) se propone ofrecer un espacio de expresión para esta población, considerando que las rutinas e imaginarios propios de la cárcel inhiben u obstaculizan estas formas de expresarse. Este espacio de trabajo y vínculo se posibilita por una demanda de los propios internos, formulada en un conversatorio desarrollado en esa cárcel en el año 2012, y que motivó el inicio de estos procesos.

Esta publicación referida se aproxima ya a su cuarto número. Los dos primeros fueron temáticamente libres; el tercero tuvo como eje el tema de la paz (relacionándose así con el proceso de negociación que en ese momento, año 2016, se estaba cerrando con éxito con la guerrilla de las Fuerzas Armadas Revolucionarias de Colombia - Ejército del Pueblo (FARC-EP). El cuarto número incluye, como novedad, una sección destinada a la población LGBTI del penal.

La asistencia regular a este sector permitió entonces conocer sus dinámicas, las expectativas y deseos de los internos que allí estudian, su visión de la institución y sus exigencias, así como la manera en que los afecta en términos de salud mental (todos estos temas, objeto de investigaciones por parte de estudiantes de la carrera mencionada). Así mismo, se tuvo acceso a programas que se implementan en este espacio, dirigidos al resto de la población carcelaria y al público externo.

El primero de ellos se llama Misión Carácter y tiene un fin resocializador, vehiculizado por medio de un programa de formación compuesto por cuatro niveles: carácter, visión, coraje y liderazgo. El segundo se denomina Delinquir no paga e intenta replicar un modelo aplicado en cárceles de Estados Unidos, dirigido a jóvenes considerado en riesgo de infringir la ley, a los que se los lleva a visitar la cárcel y, previo paso intimidante por el medio de los patios, se los expone a historias de vida de los presos, con el fin de mostrar el error cometido y sus consecuencias.

El propósito de este texto es analizar los fundamentos y objetivos del primer programa mencionado y el lugar de los participantes en el mismo, considerando los objetivos generales de reeducación social que allí se plantean y el rol general de la educación en contextos carcelarios. Para 
esto, se utilizarán las herramientas teóricas del análisis institucional (Lourau, 1970; Baremblitt, 2006) y del análisis de discurso (Fairclough, 1989; 1995; Maingueneau, 1997; Nagamine-Brandao, 1996).

\section{MARCO TEÓRICO Y METODOLÓGICO}

El análisis institucional, en sus varias expresiones teóricas, se propone analizar el funcionamiento de ciertas instituciones para entender como las prácticas y discursos que las componen representan formas posibles de alienación y opresión, que conviven con formas de organización y funcionamiento que, muchas veces de forma velada, intentan colocar esos funcionamientos en cuestión, para poder establecer nuevas praxis de carácter desalienante, capaces de expresar los deseos y demandas de los varios actores institucionales.

Esta teoría encuentra una fuerte referencia en la experiencia de Basaglia (2006), que contribuyó a acabar con la hegemonía de los modelos manicomiales en Italia. Quizás el texto que mejor expresa el sentido de esa experiencia sea La institución negada, ya que, como el propio título indica, se trató aquí de negar cualquier posibilidad de dotar a los manicomios de un carácter más humanista asumiendo, a partir del análisis de su funcionamiento, que sus prácticas y discursos eran en sí mismos patologizantes, limitados al simple control social.

En el corpus teórico del análisis institucional, se destacan algunos conceptos llave. El primero es el de institución, definida como "conjunto de prácticas sociales configuradas en la apropiación de un objeto, un determinado tipo de relación social sobre la que reivindican el monopolio, en límite con otras prácticas." (Guilhon De Albuquerque, 1980, p. 16)

De esta manera, la educación, la justicia o la familia, por ejemplo, podrían ser entendidas como instituciones. En lo que hace a este texto, las instituciones involucradas serían la justicia (en particular, en lo que hace al sistema penal) y la educación.

Las instituciones se materializan en organizaciones, como es el caso de los colegios o las universidades, en lo que hace a la educación, o de los hospitales, en el caso de la institución salud. Por su vez, cada institución u organización está compuesta por agentes institucionales, legitimados para ejercer ciertas funciones, y prácticas y discursos propias.

Para entender los procesos de continuidad o de cambio que en estos contextos se producen, se utilizan las categorías de instituido e instituyente, representando la primera a aquellas prácticas y discursos que permiten el funcionamiento inalterado de esa institución, su continuidad acrítica. El instituyente, en cambio, denomina a los procesos de transformación que pretenden alterar ese funcionamiento burocratizado, colocándolo en crisis para poder sustituirlo.

Este propósito transformador se basa, como principio ético político, en la participación y la movilización de las propias personas interesadas en esos cambios, sin ser impuestos desde fuera. Como herramienta diagnóstica, para poder entender este tipo de dinámicas institucionales, en sus formas más veladas, se utilizan las categorías de analizador y desviante. 
En el caso del analizador, se apela a alguna estrategia que el propio equipo interventor produce (un grupo focal, una obra teatral, por ejemplo) para poner en evidencia ese funcionamiento que se pretende alterar. También ese analizador puede ser natural, o sea, que los propios grupos que componen la institución expresen de alguna forma ese malestar, esa disfuncionalidad sin necesidad de un estímulo externo. El desviante refiere a ese sujeto que, con su conducta, delata también algo que la institución niega; este tipo de figuras suele ser clasificada como anómala, para negar su carácter de síntoma institucional.

El análisis de discurso analiza las condiciones sociohistóricas en las que un discurso se produce y sus formas de reproducción. En términos epistemológicos, esta teoría reúne tres campos de conocimiento: el materialismo histórico, la lingüística y la teoría del discurso. Se basa en dos conceptos principales: el de ideología, que pasa de ser entendido en el sentido marxista de falsa conciencia para más tarde comprender también una forma de vínculo (Ricoeur, 1994), y el de discurso, principalmente en la lectura foucaultiana del concepto.

La posible articulación entre estas teorías se basa en la posibilidad de analizar ciertos funcionamientos discursivos y sus efectos al interior de las instituciones, relacionándolos también con prácticas específicas y su vínculo general con el sistema social que los posibilita. De esta manera, se evita también otorgarle al discurso un carácter autónomo del marco social donde se produce o darle primacía epistemológica, en el sentido de atribuirle un papel organizador de la realidad.

En lo que hace a este trabajo en particular, estas referencias teóricas, como ya fue mencionado, se aplicarán al análisis de la denominada Misión carácter y la relación de esta con los discursos y prácticas genéricamente entendidas como resocializantes, propios de la institución carcelaria, y la forma en que las mismas se vinculan con las expectativas institucionales que la educación, en estos contextos particulares, produce.

\section{ANÁLISIS DE LA INFORMACIÓN}

La Misión carácter utiliza un documento de referencia para su trabajo, que contiene los principios y líneas fundamentales de trabajo de este programa. En el mismo se detallan los contenidos de los cuatro módulos que componen este curso, que se corresponden con cuatro principios (carácter, misión, coraje y liderazgo).

En su introducción, este texto se declara continuador de dos proyectos anteriores, denominados Proyecto compromiso y Conoce al Inpecable, destacando que está vinculado directamente al denominado tratamiento penitenciario. En particular, refiere como uno de sus propósitos modificar la cultura del aprovechamiento del otro y el relajamiento ético, afirmando valores superiores.

Estos cuatro elementos centrales mencionados contienen otros como honestidad, trabajo duro, emociones, sentido común, conflicto, ambición, perdón, liderazgo o confianza en Dios, entre otros. 
En líneas generales, este manual se asemeja a los principios de autoayuda, dado que pretende fomentar capacidades consideradas intrínsecas del sujeto, posibles de aflorar a través del esfuerzo personal y la determinación. Los varios ejemplos presentes en el texto van en el mismo sentido. Así mismo, cada tema inicia con un proverbio bíblico y el tema de cierre del curso, ya mencionado, es Confiar en Dios. La creencia religiosa se plantea como un reaseguro para evitar el egoísmo y la rebeldía y para poder hacer un uso correcto del dinero. A pesar de que ciertas condiciones sociales, como la violencia o la pobreza son mencionadas, las mismas se relacionan con algunos déficits morales, no con factores estructurales.

De esta forma, este plan de intervención y su documento de referencia conjugan dos discursos: uno de carácter marcadamente religioso y el otro impregnado de una cierta moral conservadora más genérica, posible de alcanzar por el estímulo externo y la superación individual. Estos dos discursos pueden articularse en torno al principio general de la resocialización, que supone que la cárcel puede favorecer una mayor adecuación a la norma y una integración social efectiva, más allá de las desigualdades sociales y la exclusión que exista en cada sociedad.

Los mecanismos discursivos que intentan dar carácter de verdad a las afirmaciones de ese documento son descriptos en el análisis de discurso, destacándose, por ejemplo, el del uso de la primera persona del plural, lo que crea una idea inicial de un pensamiento compartido. De esta forma, encontramos expresiones como "Vivimos en una sociedad de la rapidez" (INPEC, 2004, p. 29), "Si no intentamos que tales capacidades se desarrollen y funcionen, entonces bloqueamos nuestra propia capacidad." (INPEC, p. 31) o “Acerquémonos a Dios y aprendamos de sus principios”. (INPEC, p. 102)

La naturalización, que intenta colocar como natural un hecho socialmente producido, es otro de los recursos discursivos utilizados. Puede señalarse aquí la expresión "En cualquier sociedad hay ganadores y perdedores" (INPEC, p. 53) o "Sin patrones de moralidad que regulen el comportamiento, el autocontrol individual no existiría" (INPEC, p. 59).

Así mismo, el denominado silenciamiento funciona aquí, por medio de la negación de aspectos sociales y económicos como favorecedores de ciertas conductas.

Como ya fue dicho, estos mecanismos indicados se inscriben en una confluencia discursiva, de tono religioso - moral. La misma guarda puntos de contacto con el tratamiento moral que Pinel (1793/2003) y el alienismo en general implementaron como forma de tratamiento de la locura, en el inicio de la modernidad. En ambos casos, se trata de restituir una condición moral que devuelva a su portador la condición de ciudadanía plena.

No es casual que este tipo de proyectos se desarrolle desde el área educativa. La educación en general, en los últimos años, viene sufriendo una demanda social que exige a las instituciones educativas que operen como espacio de contención y tratamiento de problemas de diverso orden, como violencia, consumo de drogas, sexualidad, convivencia, etc. (Lajonquiere, 1970).

En el espacio particular de la cárcel, esta demanda general se articula al fin resocializador que la institución intenta sostener. Lo particular del caso es que los propios internos son convocados a 
hacer parte y sostener este tipo de proyectos, presentándose ante sus pares como una especie de referencia en este sentido.

Se debe señalar que este tipo de discursos no puede pensarse como mera imposición externa. Muchos internos manifiestan creer en los principios que este programa defiende y en la necesidad de promoverlos. No obstante, resultaría interesante acompañar la forma en que este proceso impacta en su vida cotidiana, una vez recuperada la libertad, o poder analizar si su reproducción responde de alguna manera a la exigencia institucional permanente de mostrarse en otro lugar diferente al de quien cometió el delito, sobre todo ante los ojos de la justicia. El hecho de que la participación en este programa permita acceder a descuentos en la pena, es también un incentivo innegable para incluirse en el mismo.

De esta manera, y producto de esta serie de demandas y expectativas, es posible encontrar en el área educativa de la cárcel a internos fuertemente identificados con el discurso de la institución, que con frecuencia se refieren a sus colegas presos como un otro institucional sobre el cual hay que intervenir.

En términos del análisis institucional, este tipo de acciones refuerza un instituido, que permite que las propias personas presas reproduzcan el discurso de la institución, legitimando esas prácticas institucionales y el imaginario que las permite. Así mismo, se refuerza la suposición de que cualquier conducta se basa, total o parcialmente, en una elección moral que el sujeto realiza más allá de sus condiciones de vida y contexto, liberando así a la cárcel de su responsabilidad en el aumento de la reincidencia penal y la exclusión social de las personas presas. Esta misma desresponsabilización alcanza a la sociedad en general y traza una línea imaginaria entre buenos y malos, diferenciados por esta supuesta falla moral.

El concepto de violencia simbólica de Bourdieu (1982), aplicable al contexto institucional aquí analizado, considera que la misma se inscribe en una relación asimétrica de poder, que reproduce roles sociales y estructuras mentales. En este tipo de dominación, los dominados son cómplices de la dominación sufrida, lo que favorece su perpetuación.be considerarse en primer lugar ciertas premisas éticas y política.

A pesar de este cuadro descripto, referido a un contexto particular y una práctica específica, la educación en los sistemas carcelarios puede tener otro rol, potencialmente desalienante. Para esto, más que una forma pedagógica o los contenidos curriculares adoptados, deben considerarse ciertas premisas ético-políticas, que consideren a la educación como una práctica liberadora.

En este sentido, la obra de Freire (1970) continúa siendo una referencia inevitable, desde su crítica a los modelos educativos basados en la mera transmisión de conocimientos (que este autor denomina como educación bancaria) y su reivindicación de una teoría dialógica, que permita rescatar los diversos saberes y evitar la manipulación de las clases oprimidas.

Para esto, en el ámbito carcelario en particular, debe tomar distancia de cualquier propósito relacionado con las prácticas que Zaffaroni (2011) denomina genéricamente como "re" (de 
resocialización, reinserción, etc.), para dirigirse a facilitar espacios de reflexión en torno a la situación de los sujetos privados de libertad y las causas que motivaron esta situación, tanto en términos individuales como estructurales.

También con este propósito, resulta de interés vincular la reflexión de Ranciere (2003) expresada en el texto titulado El maestro ignorante, que analiza la experiencia de Jacotot, educador convocado a dar clases sobre un tema que desconocía en un lenguaje que no manejaba. Ranciere plantea aquí un modelo de educación basado en la mutua ignorancia, donde el conocimiento se plantea como algo a producir, a partir del deseo e interés de todos los participantes de esa experiencia.

Algo similar puede pensarse en torno a la educación en las cárceles, dadas las disímiles experiencias de vida y pertenencia de clase social que suelen tener las personas involucradas en estas prácticas en esos contextos específicos. Esta relación posible, basada en el carácter democrático de la relación, constituiría un instituyente, capaz de conmover prácticas educativas tradicionales, funcionales a la institución.

Cabe mencionar que esta pretensión se instala en un escenario político en donde este tipo de propuestas encuentra un rechazo significativo, basado en la idea de que promover el pensamiento crítico y el debate en los espacios educativos significa introducir de manera artera y disimulada un propósito ideológico y político a esa práctica, aproximando la noción de ideología a cualquier pensamiento de izquierda o, inclusive, de tono liberal. Frigotto (2016) define apropiadamente este proyecto, denominado Escuela sin partido, como un intento de amordazamiento a los educadores, que se limitarían así a repetir contenidos.

No es casual que los defensores de esta forma de entender la educación apuntan a Freire como uno de sus objetivos, en tanto representa todo aquello que intentan expulsar de los ámbitos educativos. Si esta potencia freireana asusta a los grupos conservadores y reaccionarios, cuando proyectada dicha teoría y práctica en la educación común, cabe imaginar el efecto de proponer su ejercicio en los ámbitos carcelarios. Esa posible reacción y su origen es lo que hace a esa propuesta más pertinente y necesaria.

\section{CONCLUSIONES}

El análisis de la información aquí considerada, los textos que guían la denominada Misión carácter en la cárcel de Villahermosa, Cali, permiten entender como las prácticas moralistas, bajo el denominador discursivo e institucional más amplio de la resocialización, muestran facetas diversas que, en este campo particular de la educación en el ámbito carcelario, se articulan con otros discursos y prácticas próximos al tratamiento moral que guio las intervenciones de los alienistas en el inicio de la modernidad, como ya fue mencionado.

La expectativa, siempre fallida, de que alguna de estas prácticas podrá por fin permitir que las cárceles cumplan con su objetivo de articular la pena y el encierro con una futura reinserción social del 
sujeto que las sufre, alimenta la continuidad y el crecimiento de estas instituciones, así como disimula el ánimo de castigo y revanchismo social que les genera parte de su consenso.

Reiterando la referencia a la crítica estructural de Basaglia (2006) a los modelos manicomiales, es necesario apelar a ese carácter radical del cuestionamiento para afirmar que la cárcel, de la forma en la que está planteada, es una institución condenada al fracaso, al menos en lo que hace a sus propósitos formales. De esta forma, un proceso instituyente debe apuntar a negarla como institución e insertar su fracaso dentro del modelo social que permite su reproducción, señalando en este sentido a la expectativa social de segregación del mal como un mecanismo que produce consenso social para este tipo de prácticas.

Esta exigencia de eliminación de las instituciones carcelarias no debe necesariamente acompañarse de una propuesta que permita reemplazar a estos modelos punitivos, aun considerando la necesidad innegable de que cada sociedad establezca normas y formas de respuesta a su desobediencia. Esa posibilidad debe construirse a partir de un consenso y un debate abierto e implica discutir también un modelo social más incluyente.

Este momento de la realidad colombiana, donde vuelve a plantearse la discusión en torno a la posibilidad de sancionar el porte de la dosis mínima de droga (por entender que detrás de cada poseedor de droga se esconde un traficante o un enfermo), torna a este debate más urgente, dado que los delitos asociados a las drogas, en sus varias formas, han sido una de las causas principales del aumento de la población carcelaria mundial y de la ampliación de prácticas psiquiatrizantes que se inscriben en un orden similar al carcelario, en lo que hace a sus propósitos de control social y estigmatización de sectores sociales.

\section{REFERENCIAS BIBLIOGRÁFICAS}

1. BASAGLIA, Franco. La institución negada: informe de un hospital psiquiátrico. Madrid: Desván del libro, 2006.

2. BAREMBLITT, Gregorio. Compendio de análisis institucional. Buenos Aires: Madres de Plaza de mayo, 2006.

3. BOURDIEU, Pierre. Language and symbolic power. Cambridge: Harvard University Press, 1982.

4. BRAVO, Omar. Instituciones, discursos y violencia: la asociación entre locura y peligrosidad. Revista CS, v. 5, p. 241-259, 2010.

5. BRAVO, Omar. (2012). Saber, poder y salud. Apuntes desde la cárcel. In: VALERAVILLEGAS,Gregorio \&. MADRIZ, Gladys (orgs). Filosofías del buen vivir, del malvivir y otros ensayos (pp. 477 - 494). Caracas: Fondo Editorial Fundarte. 
6. BRAVO, Omar. Tratamiento penitenciario, salud mental y ciudadanía. Desafíos para la democracia y la ciudadanía. Revista CS, p. $374-368,2012^{\text {a }}$.

7. FAIRCLOUGH, Norman. Language and power. Londres: Longman Group, 1989.

8. FAIRCLOUGH, Norman. Critical discourse analysis. Londres: Longman, 1995.

9. FOUCAULT, Michel. L'Archéologie du savoir. París: Gallimard, 1969.

10. FOUCAULT, Michel. El orden del discurso. Barcelona: Tusquets, 1975.

11. FOUCAULT, Michel. Vigilar y castigar: el nacimiento de la prisión. Buenos Aires: Siglo XXI, 2002.

12. FREIRE, Paulo. Pedagogía del oprimido. Montevideo: Tierra Nueva, 1970.

13. FRIGOTTO, Gaudencio. "Escola sem partido". Imposicao de mordaca aos educadores. eMosaicos, v. 5, n 9, p. 11-13, 2016.

14. GARLAND, David. Crimen y castigo en la modernidad tardía. Bogotá: Siglo del Hombre, 2006.

15. GUILHON DE ALBUQUERUE, José Augusto. Instituição e poder: a análise concreta das relações de poder nas instituições. Rio de Janeiro: Graal, 1980.

16. GOFFMAN, Erving. Internados: nsayo sobre la situación social de los enfermos mentales. Madrid: Amorrortu, 1970.

17. LAJONQUIERE, Leandro. Infancia e ilusión (psico)-pedagógica. Buenos Aires: Nueva Visión, 1970.

18. LOURAU, René. El análisis institucional. Buenos Aires: Amorrortu, 1970.

19. MAINGUENEAU, Dominique. Novas tendências em análise do discurso. Campinas: Pontes, 1997.

20. INPEC, INSTITUTO PENITENCIARIO DE COLOMBIA. Misión carácter: sembrando semillas de libertad. Bogotá: Imprenta Nacional, 2004.

21. NAGAMINE-BRANDAO, Helene. Introdução à análise de discurso. Campinas: Unicamp, 1996.

22. PINEL, Phillippe. La mania: trattato medico-filosofico sull'alienazione mentale. Padua: Marsilio Editori, 1793/2003. 
23. RANCIERE, Jacques. El maestro ignorante: cinco lecciones sobre la emancipación intelectual. Barcelona: Laertes, 2003.

24. RICOUER, PAUL. Ideología y utopía. Barcelona: Gedisa Editorial, 1994.

\section{Omar Alejandro Bravo}

Psicólogo. Maestría y Doctorado en Psicología, Universidad de Brasilia. Posdoctorado en psicología, Universidad Estadual de Río de Janeiro.

\section{Como citar este documento:}

BRAVO, Omar Alejandro. Educacion en cárceles: práctica alienante o liberadora. Reflexão e Ação, Santa Cruz do Sul, v. 27, n. 2, abr. 2019. ISSN 1982-9949. Disponível em: <https://online.unisc.br/seer/index.php/reflex/article/view/12539>. Acesso em: doi:https://doi.org/10.17058/rea.v27i2.12539. 